\title{
GEOGRAPHIE UND SOZIOLOGIE
}

Die «junge» Wissenschaft Soziologie steht im Zuge, sich rasch zu intensivieren, was nach außen vor allem in der bereits unübersehbaren Verzweigung (Agrar- und Industriesoziologie, Familien-, Dorf-, Stadt-, Geschichts-, Kultur-, Sozial-, Kunst-,Wissenssoziologie, Pädagogische, politische, wirtschaftliche Soziologie, soziale Morphologie, Oekologie usw.) Ausdruck findet. Der Grund hiefür ist sicher nicht in der Tendenz der (oder einzelner) Soziologen zu sehen, ihre Disziplin um jeden Preis ins Rampenlicht der Öffentlichkeit zu stellen, wie manchmal beinahe zu vermuten wäre. Vielmehr ergibt er sich wohl einfach aus dem mit der unaufhaltsamen Zunahme der Bevölkerungszahl wachsenden Anfall von Sozial- bzw. Gruppenproblemen. Dieser läßt auch verstehen, wenn je länger desto vernehmlicher eine Vermehrung der Lehrstühle an den Hochschulen und überhaupt eine bessere Berücksichtigung der Soziologie an diesen gefordert wird. Wie dem nun sei: für die Geographie erwächst aus der Verstärkung der soziologischen Bestrebungen die Aufgabe, sie aufmerksam zu verfolgen. Besondern Anla $B$ hierzu hat unsere Disziplin, weil 1. ihr eigenes Objekt, die Landschaft oder die landschaftliche Erdhülle, ein ausgesprochenes «gesellschaftliches» Gebilde, ja - insofern es die «Vergesellschaftung» der Gesteine, Luftmassen, Gewässer, Pflanzen, Tiere und Menschen umfaßt - die Assoziation aller erdoberflächlichen Assoziationen darstellt (womit die Geographie gewissermaßen Soziologie der Soziologien wäre) und 2. die menschliche Gesellschaft die Landschaft so tief und nachhaltig mitprägt, daß diese letztere ohne deren Kenntnis unverständlich bliebe. In den letzten Jahren ist es denn auch beinahe zum guten Ton geworden, einerseits die älteren Geographen damit abzustempeln, da $\beta$ sie nicht'den «Weg zur Gesellschaft gefunden hätten», andererseits in fast jeder neuen geographischen Arbeit mindestens einmal das Wort Sozialgeographie oder soziologisch zu verwenden. Diese kritische Bemerkung enthebt indessen keineswegs von der Beschäftigung mit der Soziologie. Im Gegenteil, eine solche wird, wie eingangs angedeutet, je länger desto dringlicher. Wesentliche Erleichterung und Befruchtung erfuhr sie kürzlich durch zwei Bücher des Braunschweiger Geographen Prof. Dr. W. MAAS, auf die im folgenden kurz hingewiesen sei.

Das ersterschienene trägt den Titel «Geographie und Soziologie»(Braunschweig 1958, Verlag des Geogr. Instituts der Kanthochschule) und bietet, ohne das Gesamtproblem umfassend und systematisch behandeln zu wollen, in einer scheinbar losen Kapitelfolge zahlreiche wertvolle Einblicke in die Beziehungen beider Disziplinen. Dem Verfasser geht es allerdings weniger darum, wissenschaftstheoretische Betrachtungen anzustellen als vielmehr an zahlreichen Beispielen darzutun, wo und wie sich Geographie und Soziologie berühren und wie sie sich gegenseitig sachlich fördern können. Wenn hierbei auch wohl nicht mehr jeder Fachgenosse mit ihm ohne weiteres die Ansicht vertreten wird, die Geographie sei die «erklärende Beschreibung der Erdoberfläche» (vor allem weil der Terminus Erdoberfläche doch wohl zu vieldeutig ist) oder die Disziplin des «Raumerfüllenden», muß er ihm in den meisten übrigen Gedankengängen, welche der Bedeutung des Sozialen in der Landschaft gelten, weitgehend beipflichten. Augenfällig tritt sie schon im ersten Kapitel hervor. Es macht namentlich an ostmitteleuropäischen (polnischen, deutschen, tschechischen) Wald- und Industriegegenden die Wirkung von Klein- und Großgrundbesitz, Staats- und Privatwirtschaft in Forsten und Siedlungen (z. B. die konservierende Einflußnahme Preussens in Pommerellen auf die Waldflächen bis 1894, die nachmalige zerstörende der bäuerlichen Besitzer oder die Entstehung der Lodzer Textilindustrie als Folge der preußischen Zollgesetzgebung und der polnischen Regierungsbeschlüsse zur Förderung der Wirtschaft mittels Importes von Ausländern) eindrücklich. In der Folge zeigen Kapitel «die menschliche Arbeit als Zentralproblem der Anthropogeographie», «von den sozialen Gruppen», «die abnehmende Isolierung» (namentlich durch Bevölkerungszunahme, Handel, Verkehr usw.), «die gegenseitige Abhängigkeit des Menschen», «der Mensch als Faktor der physischen Geographie», «Städte», «Einflüsse von Technik, Politik und Gesetzgebung, Religion und Kunst sowie des Volkstums auf die Landschaft», zusammen mit Abschnitten über «Geophilosophie», «Geographie als Wissenschaft vom Menschen», «Psychologische Geographie» u. a. einerseits die außerordentliche Differenziertheit der Zusammenhänge zwischen Natur und menschlicher Gesellschaft. Andrerseits beleuchten sie das unauflösbare Beziehungsgefüge zwischen jener, dem Menschen und seinen sozialen, wirtschaftlichen, religiösen, technischen, politischen, künstlerischen und volkstümlichen Äußerungen, das Natur- und Kulturbetrachtung, physische, anthropische Geographie und Sozialwissenschaften insgesamt so interessant aber auch so schwer unterscheidbar macht.

Das zweite Buch von W.MAAs, «Europäisches Bauernleben einst und jetzt» (Braunschweig 1959) widmet sich «sozialgeographischen Betrachtungen» curopäischer, namentlich französischer, deutscher, englischer, spanischer und italienischer Landschaften, die allgemeine Ausführungen wie «Sozialgeographische Beobachtungen in Frankreich», «Vorläufer der Sozialgeographie im Altertum» oder «Ortsnamenkunde als Hilfswissenschaft der Sozialgeographie» einrahmen. In ihnen erweist sich der Autor, der längere Studienaufenthalte in Westeuropa wie auch im Mittelmeergebiet machen konnte und zudem Dozent in Pakistan war, als ausgezeichneter Kenner des 
französischen, englischen und westslawischen Schrifttums. Dessen eingehende Auswertung wird sowohl der deutschen Anthropogeographie als der internationalen Verständigung zweifellos gute Dienste leisten. Es sei nur auf die Aufsätze «Zur Lage der französischen Bauern», «Mönche als Waldroder in Frankreich», «die Kulturlandschaft des Bourbonnais», «Morvan 1940», «Weinbauern in Burgund», "Honoré de Balzac als Sozialgeograph», «Viermal Herbst» (ein Profil durch Frankreich und Nordwestdeutschland), «Allotments in England», «Landflucht in der Picardie» oder «Wandlungen im neumärkischen Landschaftsbild im 16. Jahrhundert» hingewiesen, um die Fülle der Aspekte anzudeuten, die gerade das zweite Buch zu einem Quell sozialgeographischer Dokumentation stempeln, wenn auch die aphoristische Form der Gedanken und nicht seltene Wiederholungen es bei aller Klarheit des Ausdrucks nicht immer leicht leserlich erscheinen lassen. (Ein weiterer Band, der sich besonders auf Mittel und Nordeuropa bezieht, ist geplant).

Die Grundgedanken beider Aufsatzsammlungen faßt wohl am besten das Schlußwort der ersten zusammen: «Die Menschen machen die Landschaften nach ihrem eigenen Bild. So wie sie sind, gestalten sie die Erde, ihre Erde (wozu freilich zu sagen wäre, daß auch sie umgekehrt von der Erde mitgeformt werden: in dem Maße wie die Europäer in den Norden der Neuen Welt hineinwuchsen, formten sie Kanada, wurden aber durch ihre Natur auch zu Kanadiern umgewandelt). Das Studium dieser Dinge ist die Anthropogeographie. Sie kann nur Sozialgeographie sein, oder anders gesagt, neben den physisch-geographischen Überlegungen müssen wir Geographen auch soziologischen Überlegungen uns hingeben. In allen Wissenschaften wendet man sich mehr und mehr dem Menschen zu, alle werden mehr humanistisch. Und die einzige Wissenschaft, die, und das seit langem, das Adjektiv menschlich zu ihrem Namen hinzusetzte: Geographie humaine, sollte sich von dieser Entwicklung ausschließen? Aber Humanismus kann nicht ein Turm aus Elfenbein sein, sondern nur die menschliche Gesellschaft, ein sozialer Humanismus, oder wenn man will ein humanistischer Sozialismus, eine Geographie nach dem Bilde des Menschen, à l'échelle humaine' (Léon Blum).» MaAs identifiziert damit also Anthropogeographie mit Sozialgeographie. Dies ist auch durchaus verständlich, wenn bedacht wird, $\mathrm{da} B$ es schwer halten würde, eine Anthropogeographie zu schaffen, die nicht Sozialgeographie wäre, weil nämlich der Mensch, der zentrale Faktor des anthropogeographischen Objekts: der menschenbelebten Landschaft oder der Kulturlandschaft, allein aus seiner elterlichen, physischen Bindung heraus ausschließlich als Gruppenwesen denkbar und daseinsfähig ist. Indessen bestehen neben dieser Auffassung Ansichten, welche die Sozialgeographie gegenüber der Anthropogeographie schlechthin inhaltlich einzuschränken suchen. Sie können sich füglich auf die Vieldeutigkeit des Grundwortes «sozial» (lat. socialis = gesellig) berufen, die auch in der Soziologie zu verschiedenen Auffassungen vom Inhalt dieser Disziplin geführt hat. Darnach erschiene Sozialgeographie mindestens in drei Bedeutungen möglich 1. als Lehre von den (menschlich-) sozialen Schichten (Klassen) in ihrer Ausprägung auf die Landschaft, 2. als Lehre von den Wirkungen menschlichen Gemeinschaften (Gruppen, Gesellschaften) überhaupt auf die Landschaft und 3.als Lehre von der menschenbelebten Erde (Anthropogeographie weitesten Sinnes wie vorhin angedeutet). Darüber hinaus wäre außerdem die Frage zu prüfen, ob der Terminus Sozialgeographie nicht in sich selbst tautologisch sei, insofern im Begriff Geographie (Gesamtgeographie, wie eingangs erwähnt) bereits und sogar in höherem Sinne (weil auch die außermenschlichen Sozietäten einschließend) das Soziale inbegriffen ist, so daß die Ableitung einer Sonderdisziplin Sozialgeographie eine untaugliche gedankliche und organisatorische Operation darstellte. Der Entscheid dieser Frage fällt deshalb nicht leicht, weil die Sozialgeographie bereits in den genannten verschiedenen Interpretationen (und wohl noch in andern) gebraucht wurde und wird. Von der wohl kaum noch diskutabeln letztgenannten Bedeutung abgesehen, läge allerdings die Verwendung Sozialgeographie $=$ Anthropogeographie (und Kulturgeographie) logisch am nächsten, wenn nicht einer «Geographie der Gemeinschaften» das unbedingt notwendige Korrelat einer Geographie der Persönlichkeit(en) gegenübergestellt wird. Doch muß die Zukunft weisen, ob logischen oder «praktischen» Überlegungen gefolgt wird. Da $\beta$ MaAs sich für eine «weitsinnige» Sozialgeographie eingesetzt hat, mu $\beta$ ihm jedenfalls als Positivum angerechnet werden. Freilich finden sich im Verlauf seiner Gedankengänge auch solche, aus denen auf eine engere Auslegung geschlossen werden könnte, so wenn er z. B. (1959, p. 7$)$ die Sozialgeographie als Lehre definiert, "wie die in verschiedene soziale Gruppen (Klassen, Völker, Religionen, Kulturkreise) zerfallende Menschheit je nach ihrer Gruppe die Landschaft anders umgestaltet». Doch kann hier raumeshalber nicht auf solche Varianten eingegangen werden. Der Nachdruck seiner beiden Bücher liegt ja, wie betont, nicht in methodologisch-erkenntnistheoretischen Auseinandersetzungen, sondern in dem im ganzen durchaus überzeugenden, weil an zahlreichen Exempeln geführten Nachweis von der tiefgreifenden und globalen Umprägung der Landschaft durch den Menschen als Gruppenorganismus. Just er und die besondere Form, die ihm der Autor gegeben hat, dürfte geeignet sein, auch die Soziologen davon zu überzeugen, $\mathrm{da} \beta$ sie ohne sozialgeographische Methodik und Denkweise ihre eignen Aufgaben - die Problematik der menschlichen Gruppen - nicht lösen können, daß sie vielmehr deren landschaftliche Verhaftung je länger desto bewußter und systematischer in ihre Erkenntnisarbeit einbeziehen müssen. Damit ist der Grundlegung einer systematischen Geo- 
soziologie oder geographischen Soziologie als Lehre von der Wirkung der Landschaft auf die Gesellschaft ebenso der Weg klar gewiesen wie einer Sozialgcographic, in deren Erkenntniszentrum die «gesellschaftlich» gestaltete Landschaft steht. Es darf gehofft werden, da $B$ beide Wissenschaftsgruppen auf der Basis der Einsicht in diese grundsätzliche Scheidung der Aspekte gemeinsam die bestmögliche Förderung ihrer Erkenntnisse ziehen werden.

E. W.

\section{DIE SAMMLUNG FÜR VÖLKERKUNDE DER UNIVERSITÄT ZÜRICH IM JAHRE 1959}

Während die Sammlungsberichte bisher gleichzeitig mit dem im Laufe des Monats April zu Ende gehenden Geschäftsjahr der G. E. G.Z. abgeschlossen wurden, werden sich die Jahresberichte der Sammlung für Völkerkunde in Zukunft einfachheitshalber auf das Kalenderjalrr beziehen. Einige unvermeidliche Ueberschneidungen mit den Angaben des letzten Jahresberichts bitten wir zu entschuldigen.

Personal und freizuillige Mitarbeiter. Wie bisher wurden die laufenden Arbeiten in der Sammlung durch die beiden Konservatorinnen mit halbtägiger Verpflichtung, Frl. Dr. E. Leuzinger und Frl. G. Wil.DBERGER, besorgt. Im Rahmen des 600-Stundenkredits waren freiwillig als Hilfskräfte verpflichtet: Frl. cand. phil. I. Ariane Rump, dazu ab September Frl. Dr. Eva Stoll. Wie letztes Jahr stellten sich auch für 1959 die Damen E. ZINK und Dr. A. GARBADE freiwillig für die Beschriftung von Museumsobjekten und für die Katalogisierung von Zeitschriften und Büchereingängen zur Verfügung.

Bauliches, Mobiliar, Organisation und Verzaltung. Im Laufe des Jahres wurden der Arbeitsraum der Konservatorinnen sowie Vorraum und Büro des Vorstehers renoviert, neu gestrichen und mit Neonbeleuchtung versehen. Versuchsweise wurde die Ceylon-Vitrine im Indiensaal mit Neon-Innenbeleuchtung ausgestattet. Für 1960 ist die Einrichtung von Neon-Beleuchtung für alle Abteilungen und für die meisten Vitrinen vorgesehen. Das kantonale Hochbauamt stellte der Sammlung eine

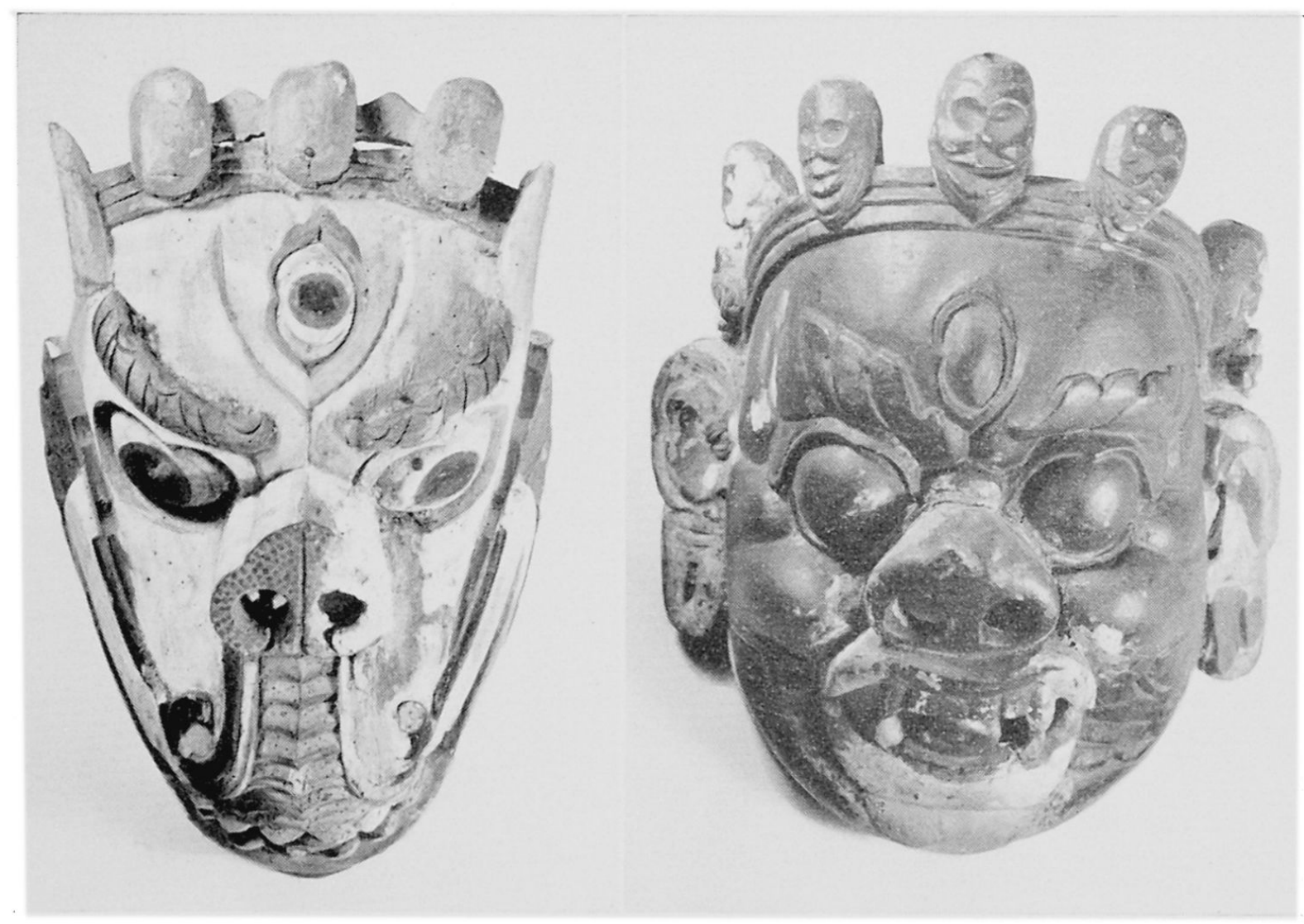

Fig. 1 (links) Nepalesische Löwenmaske "Singhdong Ma», weiß, (Kat. No. 12290), $35 \times 27,5 \mathrm{~cm}$. Sammler: Arthur Dürst. - Fig. 2 (rechts) Nepalesische Maske "Gombo Tschaktschi” aus dem Kloster Samdsche Tortscheda, dunkelblau (Kat. No. 12291), 35 × 28 cm. Sammler: Arthur DürsT. Geschenk der Schweiz. Stiftung für alpine Forschung.

Photos: G. Wildberger 